\title{
Intermittent Transition to Turbulence in Dissipative Dynamical Systems
}

\author{
Yves Pomeau and Paul Manneville* \\ Commissariat à l'Énergie Atomique, Division de la Physique, Service de Physique Théorique, \\ F-91190 Gif-sur-Yvette, France
}

\begin{abstract}
We study some simple dissipative dynamical systems exhibiting a transition from a stable periodic behavior to a chaotic one. At that transition, the inverse coherence time grows continuously from zero due to the random occurrence of widely separated bursts in the time record.
\end{abstract}

\section{Introduction}

A number of investigators [1] have observed in convective fluids an intermittent transition to turbulence. In these experiments the external control parameter, say $r$, is the vertical temperature difference across a Rayleigh-Bénard cell. Below a critical value $r_{T}$ of this parameter, measurements show well behaved and regular periodic oscillations. As $r$ becomes slightly larger than $r_{T}$ the fluctuations remain apparently periodic during long time intervals (which we shall call "laminar phases") but this regular behavior seems to be randomly and abruptly disrupted by a "burst" on the time record. This "burst" has a finite duration, it stops and a new laminar phase starts and so on. Close to $r_{T}$, the time lag between two bursts is seemingly at random and much larger than - and not correlated to - the period of the underlying oscillations. As $r$ increases more and more beyond $r_{T}$ it becomes more and more difficult and finally quite impossible to recognize the regular oscillations (see Fig. 1).

This sort of transition to turbulence is also present in simple dissipative dynamical systems [2] such as the Lorenz model [2a]. We present here the results of some numerical experiments on this problem.

When a burst starts at the end of a laminar phase this denotes an instability of the periodic motion due to the fact that the modulus of at least one Floquet multiplier [3] is larger than one. This may occur in three different ways: a real Floquet multiplier crosses the unit circle at $(+1)$ or at $(-1)$ or two complex conjugate multipliers cross simultaneously. To each of these three typical crossings we may associate one type of intermittency that we shall call for convenience type

* DPh. G. PSRM, Cen Saclay, Boîte Postale 2, F-91190 Gif-sur-Yvette, France 

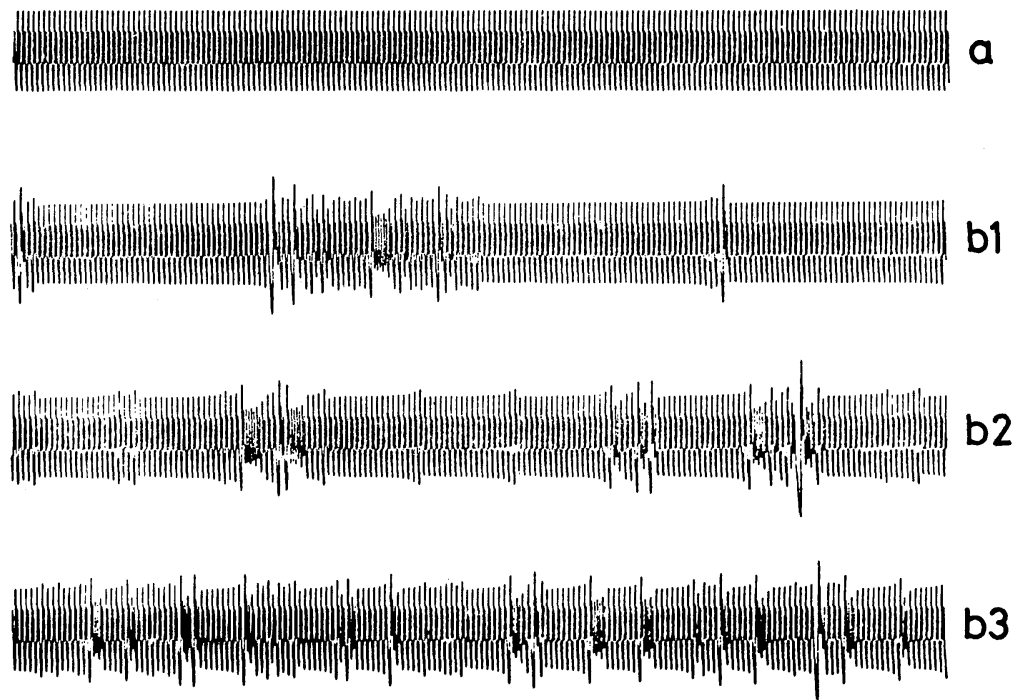

Fig. 1a and b. Time record of one coordinate $(z)$ in the Lorenz model. a Stable periodic motion for $r=166$. b Above the threshold the oscillations are interrupted by bursts which become more frequent as $r$ is increased

1 : crossing at $(+1)$; type 2 : complex crossing; and type 3 : crossing at $(-1)$ respectively. In all these three cases our numerical studies show that the Lyapunov number grows continuously from zero beyond the onset of turbulence. In what follows we shall present some simple estimates for the "critical behavior" of this Lyapunov number in the vicinity of the turbulence threshold and compare them with the results of numerical experiments.

\section{Type 1. Intermittency in the Lorenz Model}

The Lorenz system reads [4]:

$$
\frac{d x}{d t}=\sigma(y-z) ; \quad \frac{d y}{d t}=-x z+r x-y ; \quad \frac{d z}{d t}=x y-b z,
$$

where $\sigma, b$, and $r$ are parameters. We have kept $b$ and $\sigma$ fixed at their original values $(\sigma=10, b=8 / 3)$. Integrating system (1) around $r=166$ one finds for $r$ slightly less than $r_{T}(\simeq 166.06)$ regular and stable oscillations for a random choice of initial condition (Fig. 1a). For $r$ slightly larger than $r_{T}$ these oscillations are interrupted by bursts (Fig. 1b). This can be explained quite simply by studying the Poincaré map (restricted here to be 1-dimensional without loss of significance). Let $f$ be the function such that $y_{n+1}=f\left(y_{n}, r\right)$ where $y_{n}$ is the $y$-coordinate of the $n^{\text {th }}$ crossing of the plane $x=0$. Near $r=r_{T}$ the curve of equation $y^{\prime}=f(y, r)$ is nearly tangent to the first bissectrix (Fig. 2). For $r$ slightly below $r_{T}$, this curve has two intersections with the bisectrix, they collapse into a single point at $r=r_{T}$ while for $r>r_{T}$ the curve is lifted up and no longer crosses the first bisectrix so that a "channel" appears between them (Fig. 3). Hence the successive iterates generated by the map 


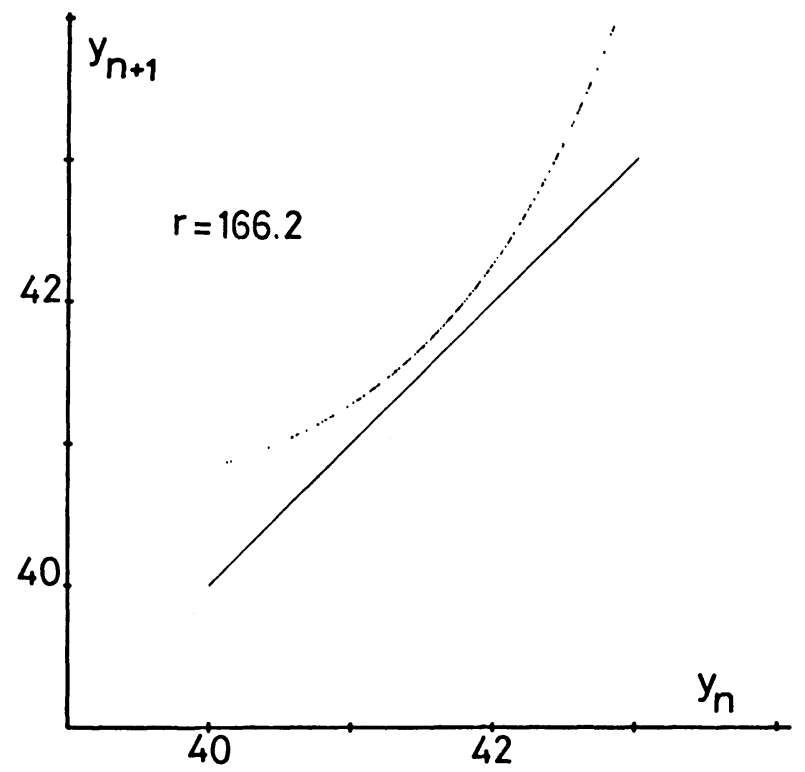

Fig. 2. A part of the Poincaré map along the y-coordinate for $r=166.2$ slightly beyond the intermittency threshold $\left(r_{T} \simeq 166.06\right)$

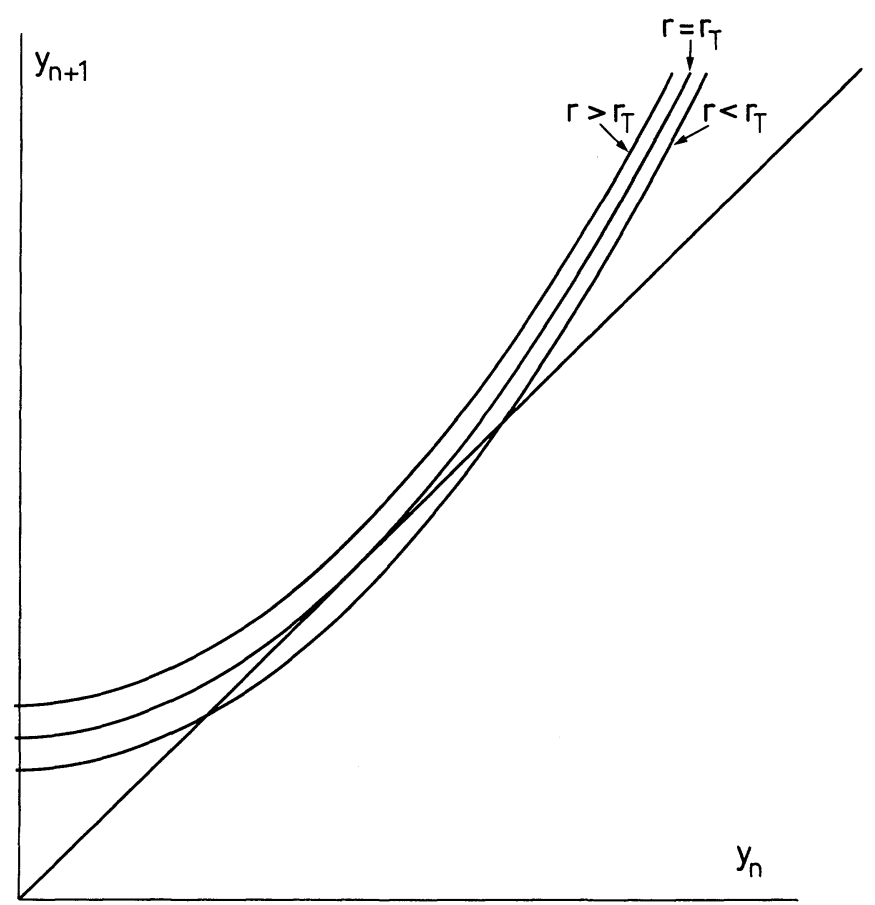

Fig. 3. Idealized picture of the deformation of $y_{n+1}\left(y_{n}\right)$ explaining the transition via intermittency. For $r<r_{t}$ two fixed points coexist one stable the other unstable. They collapse at $r=r_{T}$ and then disappear leaving a channel between the curve and the first bisectrix 


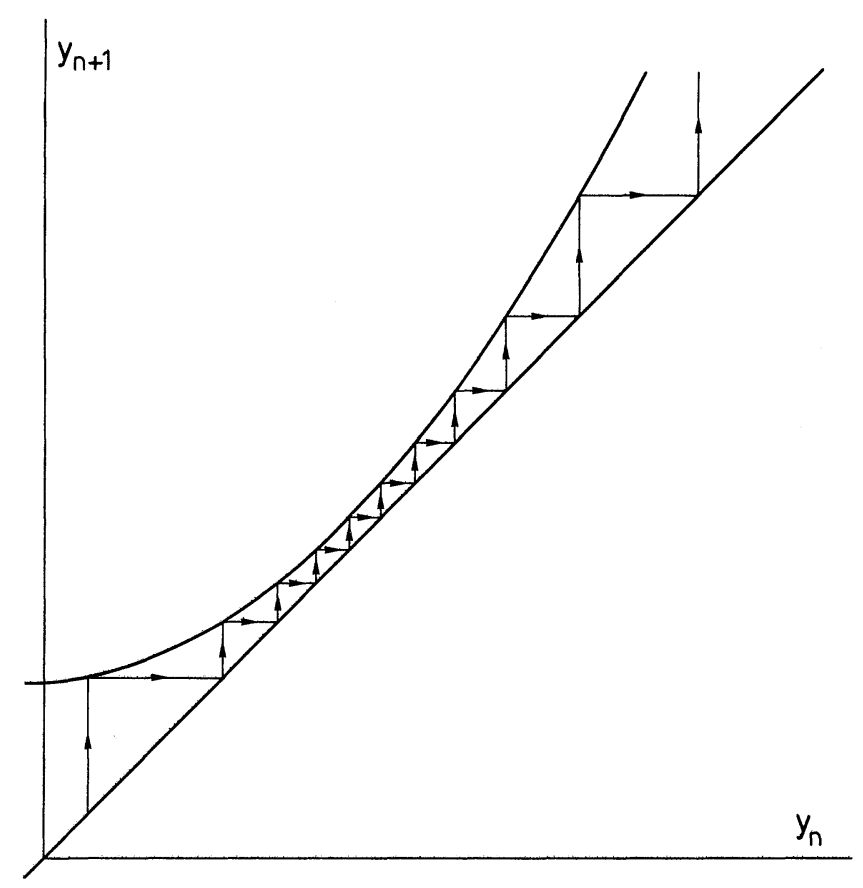

Fig. 4. The motion through the channel corresponds to the laminar phase of the movement. The slow drift is quite imperceptible on the time record of Fig. 1b

$y \rightarrow f(y, r)$ travel along this channel, which requires a large number of iterations (Fig. 4). To estimate this number let us consider a "generic form" for $f(y, r)$ in the region considered:

$$
f(y, r)=y+\varepsilon+y^{2} \text { (+ higher order terms - H.O.T.), }
$$

where $\varepsilon=\left(r-r_{T}\right) / r_{T}$. Near $\varepsilon=0_{+}$the difference equation

$$
y_{n+1}=y_{n}+\varepsilon+y_{n}^{2} \text { (+ H.O.T.) }
$$

can be approximated by a differential equation over $n$ and an elementary estimate shows that a number of iteration of the order of $\varepsilon^{-1 / 2}$ is needed to cross the channel. This is in nice agreement with our numerical simulation of system (1) (Fig. 5). After each transfer the burst destroys the coherence of the motion. This leads one to conclude that near $\varepsilon=0_{+}$the Lyapunov number varies as $\varepsilon^{1 / 2}$. Though this is consistent with our first numerical estimates, close to the intermittency threshold the Lyapunov number converges so slowly that it is difficult to get with precision, so we have preferred to turn to a modelling of the Poincaré map. We have got a qualitatively similar behavior for the following map of $S^{1}=[0,1[$

$$
\theta \rightarrow 2 \theta+r \sin 2 \pi \theta+0.1 \sin 4 \pi \theta(\bmod 1) .
$$

As shown in Fig. 6 this applies $S^{1}$ twice on itself and it is intermittent at $r_{T} \simeq-0.24706$. In this model, as well as those we shall consider later, the 


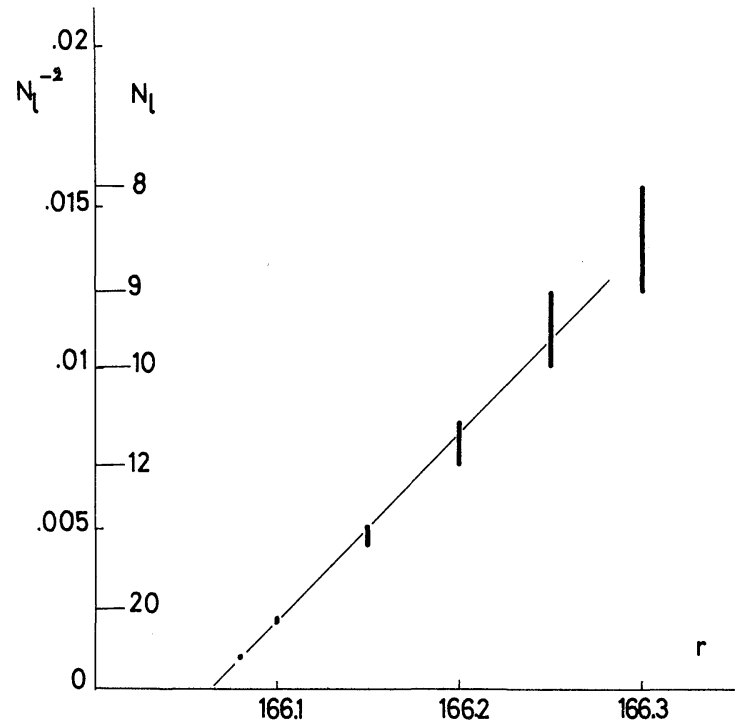

Fig. 5. The square of $N_{l}$ the largest number of cycles during a laminar period is inversely proportional to the distance from the threshold $r-r_{T} . N_{l}$ is given within 1 cycle to account for the uncertainty in the definition of the beginning/end of a laminar phase

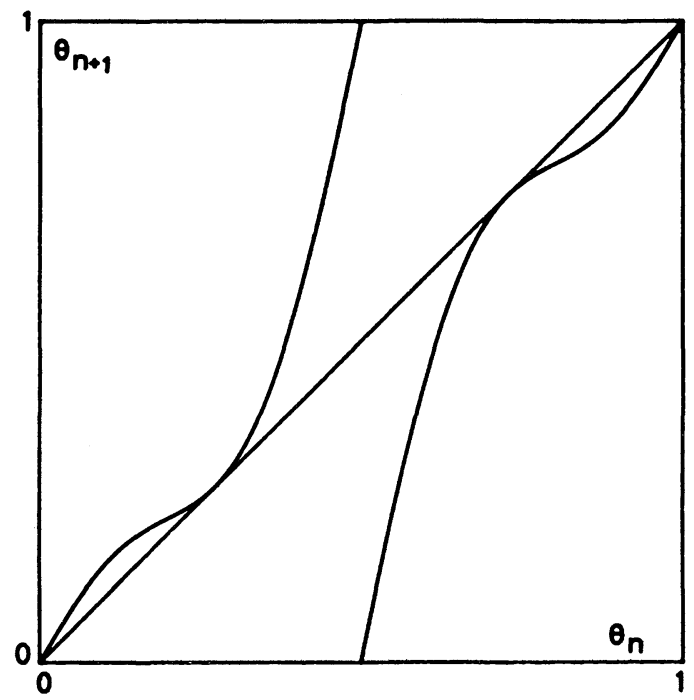

Fig. 6. Model mapping displaying qualitatively the same behavior as the Lorenz model around $r=166$

possibility of starting a laminar phase after a burst comes from the fact that the map is not invertible. In diffeomorphisms the "relaminarization" cannot occur in this way due to the uniqueness of preimages. However dynamical systems for which the "reduced" Poincare map takes a form similar to (2) [and later to (3) or (5)] can be constructed simply by adding other dimensions along which fluc- 


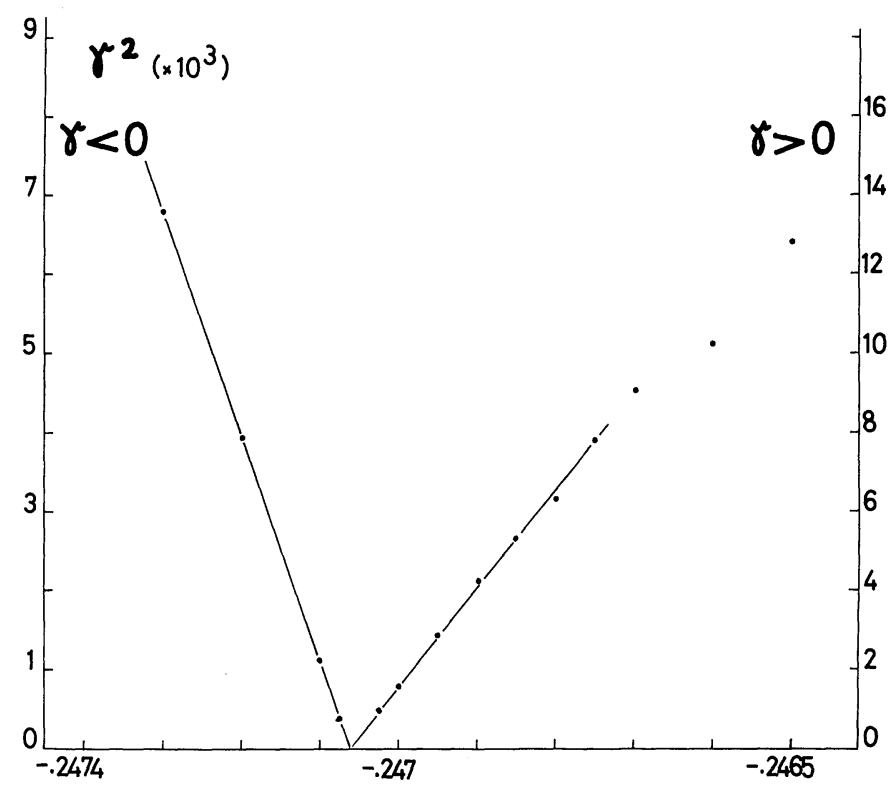

Fig. 7. For the model mapping $r_{T} \simeq-0.24706$. For $r<r_{T}$ the Lyapunov number $\gamma$ is negative and varies as $-\sqrt{r_{T}-r}$ while for $r>r_{T}$ it is positive and grows like $\sqrt{r-r_{T}}$

tuations are stable [5]. Numerical simulation of the model defined by (2) can easily be performed using a desk-top computer. As expected the Lyapunov number grows with the 1/2-power near the threshold (Fig. 7).

\section{Type 2. Intermittency}

In order to study numerically this case we have considered a map that applies the torus $T^{2}=[0,1[\times[0,1[$ four times onto itself: in complex notations $z=x+i y$

$$
\begin{aligned}
& z^{\prime}=\lambda z+\mu|z|^{2} z \text { close to the origin }(\lambda \text { complex and } \mu \text { real }), \\
& z^{\prime}=2 z \text { far from the origin }
\end{aligned}
$$

with a smooth interpolation inbetween. The fixed point $z=0$ of this map looses its stability when the complex parameter $\lambda$ crosses the unit circle, the coefficient $\mu$ of the cubic term being so chosen as to avoid the appearance of a stable limit cycle or equivalently to make the bifurcation subcritical i.e. $\mu=\mu \operatorname{Re}\{\lambda\}>0$. Iterations of the above map show intermittency when $|\lambda|=1+\varepsilon$ and $\varepsilon \rightarrow O_{+}$. Once an iterate falls near $z=0$, it enters a laminar phase and a large number of further iterations are needed to expell it towards the "bursting region" (where correlations are broken) defined by $|z|>\varrho^{*}, \varrho^{*}$ being fixed and $\varepsilon$-independent, roughly in the interpolating region. To find how the Lyapunov number grows near the intermittency threshold one may reason as follows: Let $\varrho_{j}=\left|z_{j}\right|$ be the distance of the $j^{\text {th }}$ iterate to the fixed point. The iterates rotate around the fixed point due to the complex nature of $\lambda$ but we shall neglect the angular variation and only consider the growth of the modulus 


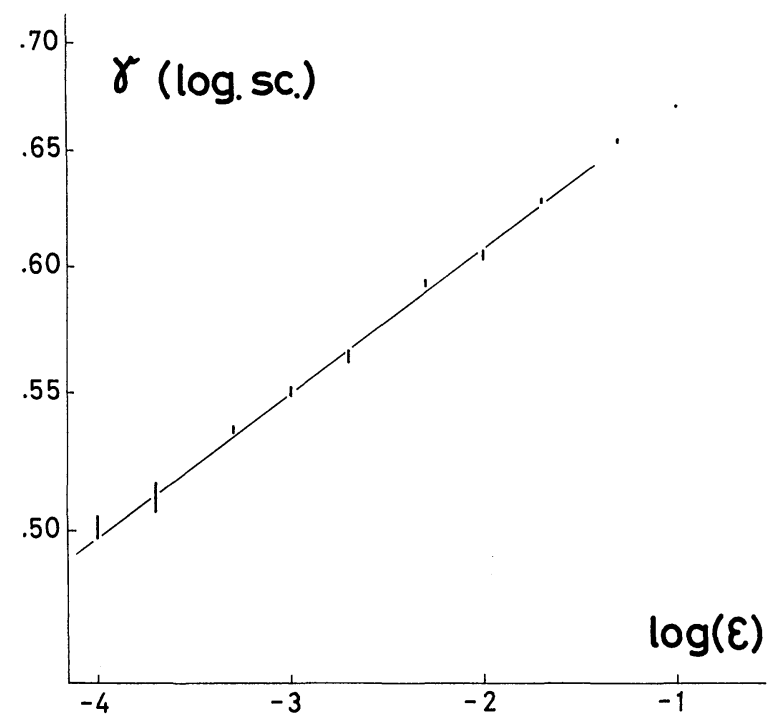

Fig. 8. On the torus $T_{2}$ for $\lambda=(1+\varepsilon) \exp i \varphi, \varphi=0.05 \mathrm{rd}$ and $\mu=20$ "mean field" theory predicts $\gamma \sim \ln (1 / \varepsilon)$ while the numerical simulation gives $\gamma \sim \varepsilon^{\alpha}$ with $\alpha \sim 0.04$

$\varrho$. Near $\varrho=0$ it is approximatively given by

$$
\varrho_{n+1}=(1+\varepsilon) \varrho_{n}+\bar{\mu} \varrho_{n}^{3}+\text { H.O.T. }
$$

Now let us examine a laminar cycle with starting point at $\varrho=\tilde{\varrho} \ll \varrho^{*}$. If $\tilde{\varrho} \gg \varepsilon^{1 / 2}$ one easily sees that a number of iteration of order $1 / \varrho^{2}$ are needed to reach $\varrho^{*}$ and enter a turbulent burst. On the other hand if $\tilde{\varrho} \ll \varepsilon^{1 / 2}$ the laminar cycle ends after $\varepsilon^{-1} \ln \varrho$ iterations approximately. Assuming then that $\tilde{\varrho}$ is at random with probability $\tilde{\varrho} d \varrho$ in the circle of center 0 and radius $\varrho^{*}$ the estimates given above yield $\ln (1 / \varepsilon)$ as an order of magnitude for both the mean duration of a laminar period and the inverse Lyapunov number near $\varepsilon=0_{+}$. This is in slight disagreement with our computer experiments which seem to indicate rather a power-like growth of the Lyapunov number $\gamma \sim \varepsilon^{\alpha} \alpha$ small and positive (Fig. 8). This descrepancy between the naive theory presented above and computer results may come from the neglect of fluctuations about the mean length of the laminar cycles, which makes the procedure used sound much like a "mean field theory" in the usual jargon of phase transitions (it may also come from the neglect of the rotation of iterates affecting the statistics in an unknown way).

\section{Type 3. Intermittency}

The last type of intermittency we shall examine may occur when the Floquet multiplier is real and crosses the unit circle at $(-1)$. Although a differential system has been found which displays this kind of behavior [2b] we shall report here on the simulation of the following mapping of the circle $S_{1}$ onto itself:

$$
\theta \rightarrow 1-2 \theta-\frac{1}{2 \pi}(1-\varepsilon) \cos \left[2 \pi\left(\theta-\frac{1}{12}\right)\right](\bmod 1) \text {. }
$$




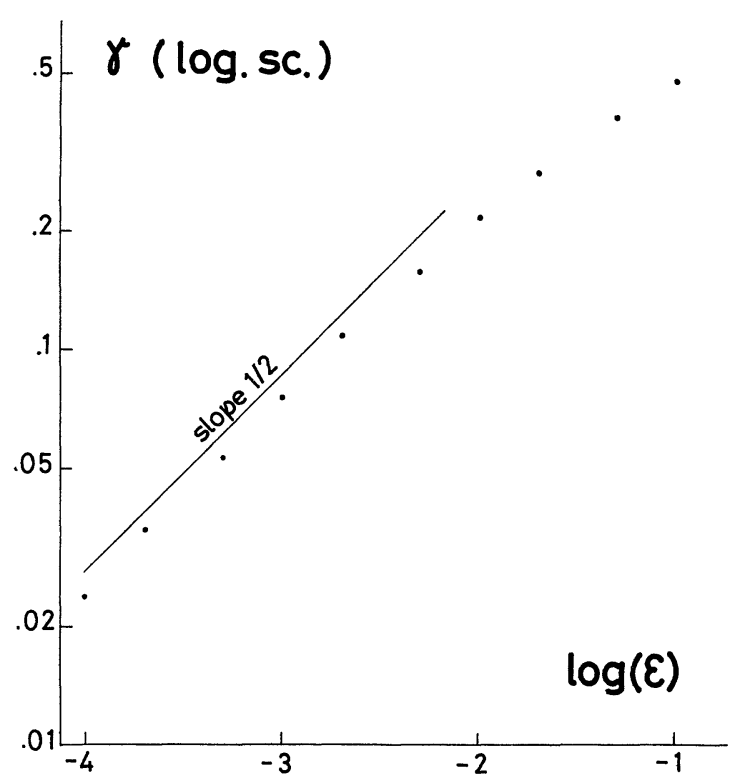

Fig. 9. On the torus $T_{1}$ for type 3 intermittency the Lyapunov number $\gamma$ grows as $\varepsilon^{1 / 2}$

This map applies $S^{1}$ twice onto itself and it reverse the orientation so that the eigenvalue of the map linearized near the fixed point $\theta_{F}=1 / 3$ can easily be made negative. Near the fixed point the map expands as

$$
\bar{\theta}_{n+1}=-(1+\varepsilon) \bar{\theta}_{n}-\frac{(2 \pi)^{2}}{6} \bar{\theta}_{n}^{3}+\text { H.O.T. }\left(\bar{\theta}=\theta-\theta_{F}\right) .
$$

The most general form would be

$$
\bar{\theta}_{n+1}=-(1+\varepsilon) \bar{\theta}_{n}+a \theta_{n}^{2}+b \theta_{n}^{3},
$$

$a$ and $b$ being constant. If the r.h.s. of (7) has a positive Schwarzian derivative that is here $b+a^{2}<0$ then the bifurcation at $\varepsilon=0$ is subcritical and type 3 intermittency can occur. This is precisely the case with (5) since $a=0$ and $b<0$. To estimate the mean length of a laminar phase one considers instead of (6) or (7) the equation giving $\bar{\theta}_{n+2}$ in function of $\bar{\theta}_{n}$. This relation is basically of the same form as Eq. (4) (quadratic terms vanish at $\varepsilon=0$ and are in inessential for $\varepsilon$ small enough). Thus one reasons as for type 2 intermittency with the difference that now the problem is strictly unidimensional so that the probability measure for for the starting point of

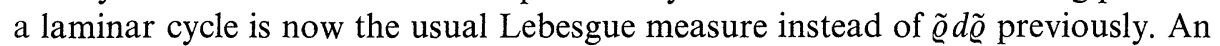
elementary calculation shows that the Lyapunov number should grow like $\varepsilon^{1 / 2}$ near threshold, this time in agreement with the computer experiment (Fig. 9).

\section{Conclusion}

Intermittency is a quite common phenomenon in experimental turbulence. The theory sketched in this paper is more especially related with the case of convection in confined geometries [1] but intermittency is also well known in boundary layers 
and pipe flows [6] and even in $1 / f$ - noise theory [7]. Despite the different meanings of the term "intermittency", the possibility remains that the kind of dynamics described by the models we have studied could afford a qualitative understanding of all these phenomena.

\section{References}

1. Maurer, J., Libchaber, A., Bergé, P., Dubois, M.: Personal communications

2. (a) Manneville, P., Pomeau, Y.: Phys. Lett. 75A, 1 (1979)

(b) Arneodo, A., Coullet, P., Tresser, C.: Private communication

3. Iooss, G. : Bifurcation of maps and applications. In: North-Holland Math. Studies 36. Amsterdam, New York: North-Holland 1979

4. Lorenz, E.N.: J. Atmos. Science 20, 130 (1963)

5. Pomeau, Y.: Intrinsic stochasticity in plasmas, Cargèse 1979. (eds. G. Laval, D. Gresillon). Orsay: Editions de Physique 1979

6. Tritton, D.J.: Physical fluids dynamics. New York: Van Nostrand-Reinhold 1977

7. Mandelbrot, B. : Fractals form chance and dimension. San Francisco: Freeman 1977

Communicated by D. Ruelle

Received January 17, 1980 
